Research Article

\title{
Spacelike Sweeping Surfaces and Singularities in Minkowski 3-Space
}

\author{
Fatemah Mofarreh $\left(\mathbb{D},{ }^{1}\right.$ Rashad A. Abdel-Baky ${ }^{D},{ }^{2}$ and Nadia Alluhaibi ${ }^{3}{ }^{3}$ \\ ${ }^{1}$ Mathematical Science Department Faculty of Science, Princess Nourah Bint Abdulrahman University, \\ Riyadh 11546, Saudi Arabia \\ ${ }^{2}$ Department of Mathematics, Faculty of Science, University of Assiut, Assiut 71516, Egypt \\ ${ }^{3}$ Science and Arts College, Rabigh Campus, King Abdulaziz University, Jeddah, Saudi Arabia \\ Correspondence should be addressed to Fatemah Mofarreh; fyalmofarrah@pnu.edu.sa
}

Received 31 May 2020; Revised 23 December 2020; Accepted 12 January 2021; Published 30 January 2021

Academic Editor: Alessandro Gasparetto

Copyright (C) 2021 Fatemah Mofarreh et al. This is an open access article distributed under the Creative Commons Attribution License, which permits unrestricted use, distribution, and reproduction in any medium, provided the original work is properly cited.

\begin{abstract}
We considered the spacelike sweeping surface with rotation minimizing frames at Minkowski 3-space $\mathbb{E}_{1}^{3}$. We presented the new geometric invariant to demonstrate geometric properties and local singularities for this surface. Then, we derived sufficient and necessary conditions of the surface to become developable ruled surfaces. Additionally, its singularities are studied. Finally, examples are illustrated to explain the applications of the theoretical results.
\end{abstract}

\section{Introduction}

The vision of the computer is the automatical analysis of image sequences in order to build the 3-dimensional surface form. Recently, various majors of mathematics are used for computer vision and medical imaging. Projective geometry, as an old mathematical subject, still used to characterize connections between both lines and points in several images to the same theme. In addition, differential geometry is used to characterize the shape of the curve and surface in engineering. Both Rene Thom, French mathematician, and Hassler Whitney introduced some developments in mathematical thinking and methods, especially the concept of singularity theory that contains catastrophes and bifurcations. Singularity theory now as the direct application of differential calculus is important to gain vital results in many subjects as computer vision and medical imaging (e.g., [1-7]).

A canal surface is the surface that can be generated by the one-parameter set of spheres determined by a radius function and center curves: in case a radius function is a constant function, the canal surface is the envelope of the moving sphere and named the sweeping surface. Some well- known examples of the sweeping surface are circular cylinder and circular cone (radius of spheres is not constant), surface of revolution, and Dupin cycloids. More specific, the sweeping surface named the tubular surface in case the radius of the generating spheres is constant. Sweeping surfaces are very important for descriptive geometry, especially for solid and surface modeling at computer-aided design, computer-aided manufacturing (CAD/CAM), and the design of trajectory movement for robots [8-14]. It is a fact that a sweeping surface can be a developable surface. The developable surface defines the surface that can become unfolded (or developed) to the plane with the absence of any stretch or tear. As known at differential geometry, with considering sufficient differentiability, the developable surface defines the plane, conical surface, cylindrical surface, or tangent surface of the curve or the structure of one of those kinds. Therefore, the developable surface is considered as the ruled surface, such that every point at the same ruling shares the common tangent plane. The rulings are principal curvature lines which vanish normal curvature and Gaussian curvature that is vanishing at every point. As a result, the developable surface is a significant surface in (CAD/CAM) 
and geometric modeling as it is used for motion analysis or designing cars and ships [15-19].

The essential tool to analyze the curve and surface at differential geometry is the Serret-Frenet frame that is the most used frame at Euclidean 3-space and Minkowski 3space [16-20]. The main apparatus in the previous literatures are Serret-Frenet formulas and some linked functions on the curve as a distance-squared function in addition to the height function. Based on Serret-Frenet formulas, the singularity of those functions can be studied from the view of extrinsic differential geometry. Because the Serret-Frenet will not be defined everywhere, there is a need for a new mathematical tool to be used for analysis purposes. In [20], Bishop gave the alternative moving frame to points on the curve at Euclidean 3-space using parallel vector fields. It named rotation minimizing frame (RMF) or Bishop frame of the curves [19-21]. Analogous to Bishop frame in Euclidean 3-space, there is a similar Lorentzian frame which named Lorentzian Bishop frame, constructed along the curve at Lorentzian space, and it is the analog of the Bishop type frame as applied to Lorentzian geometry. At Lorentzian space, using Minkowski Bishop frame through the curve as a basic tool is preferred than using Serret-Frenet frame [21-23].

In fact, there is no more literature review regarding singularities of sweeping surfaces relating to the Minkowski Bishop frame. Therefore, this study aims to cover some needs, where it is inspired by the study of Izumiya et al. [7] and Bishop [19]. At this study, we establish the Lorentzian Bishop frame along the unit speed spacelike curve with timelike principal normal and develop the local differential geometry of spacelike sweeping surfaces at Minkowski 3space. Using unfolding theory at singularity theory in this study, generic singularities of this sweeping surface are classified. A new invariant relating to singularities of this sweeping surface is presented. It is founded that generic singularities of this sweeping surface are cuspidal edge and swallowtail, and these kind of singularities can be characterized by this invariant, in the same order. Afterward, we solved this problem of requiring the surface which is the spacelike sweeping surface and, at the same time, the spacelike developable surface. Some examples are introduced in order to demonstrate theoretical results.

\section{Preliminaries}

Some definitions and basic concepts are given which will be used (for instance $[8,24,25]$ ). Suppose $\mathbb{E}_{1}^{3}$ is 3 -dimensional Minkowski space, the 3 -dimensional real vector space $\mathbb{R}^{3}$ considers the metric

$$
<d \mathbf{r}, d \mathbf{r}>=d r_{1}^{2}+d r_{2}^{2}-d r_{3}^{2},
$$

where $\left(r_{1}, r_{2}, r_{3}\right)$ denotes the canonical coordinates in $\mathbb{R}^{3}$. Any vector $\mathbf{r}$ of $\mathbb{E}_{1}^{3}$ named spacelike in case $\langle\mathbf{r}, \mathbf{r} \gg 0$ or $\mathbf{r}=0$, timelike in case $\langle\mathbf{r}, \mathbf{r}\rangle\langle 0$, and lightlike or null in case $\langle\mathbf{r}, \mathbf{r}\rangle=0$ and $\mathbf{r}=0$. The timelike or lightlike vector at $\mathbb{E}_{1}^{3}$ is named causal. Also, with the norm $\|\mathbf{r}\|=\sqrt{|\langle\mathbf{r}, \mathbf{r}\rangle|}$, the vector $\mathbf{r}$ is the spacelike unit vector if $\langle\mathbf{r}, \mathbf{r}\rangle=1$ and a timelike unit vector if $\langle\mathbf{r}, \mathbf{r}\rangle=-1$. Therefore, we say that a smooth map $\beta: I \longrightarrow \mathbb{E}_{1}^{3}$ is spacelike, timelike, or lightlike, if its velocity vector $\beta^{\prime}$ is spacelike, timelike, or lightlike, in the same order. Similarly, the surface is named spacelike, timelike, or lightlike if its tangent planes are spacelike, timelike, or lightlike, respectively. For any two vectors $\mathbf{r}, \mathbf{p} \in \mathbb{E}_{1}^{3}$, the inner product is a real number $\langle\mathbf{r}, \mathbf{p}\rangle=-r_{1} p_{1}+r_{2} p_{2}+r_{3} p_{3}$, and the vector product is given as

$$
\begin{aligned}
\mathbf{r} \times p= & \left|\begin{array}{ccc}
\mathbf{f}_{1} & \mathbf{f}_{2} & -\mathbf{f}_{3} \\
r_{1} & r_{2} & r_{3} \\
p_{1} & p_{2} & p_{3}
\end{array}\right|=\left(\left(r_{2} p_{3}-r_{3} p_{2}\right),\left(r_{3} p_{1}-r_{1} p_{3}\right),\right. \\
& \left.-\left(r_{1} p_{2}-x_{2} p_{1}\right)\right)
\end{aligned}
$$

where $\mathbf{f}_{1}, \mathbf{f}_{2}, \mathbf{f}_{3}$ are the canonical bases of $\mathbb{E}_{1}^{3}$. For a fixed point $\mathbf{p} \in \mathbb{E}_{1}^{3}$ and the positive number $c>0$, hyperbolic and Lorentzian (de Sitter space) spheres, in the same order, are given as

$$
\begin{aligned}
& \mathbb{H}_{+}^{2}(\mathbf{p}, c)=\left\{\mathbf{a} \in \mathbb{E}_{1}^{3} \mid\langle\mathbf{a}-\mathbf{p}, \mathbf{a}-\mathbf{p}\rangle=-c^{2}\right\} \\
& \mathbb{S}_{1}^{2}(\mathbf{p}, c)=\left\{\mathbf{a} \in \mathbb{E}_{1}^{3} \mid\langle\mathbf{a}-\mathbf{p}, \mathbf{a}-\mathbf{p}\rangle=c^{2}\right\}
\end{aligned}
$$

We define

$$
\mathbb{L} \mathbb{C}_{p}^{*}=\left\{\mathbf{a} \in \mathbb{E}_{1}^{3} \mid\langle\mathbf{a}-\mathbf{p}, \mathbf{a}-\mathbf{p}\rangle=0\right\}-\{\mathbf{p}\},
$$

and it is called the (open) lightcone at the vertex $\mathbf{p}$. In case $\mathbf{p}=0$ and $c=1$, we define $\mathbb{L} \mathbb{C}_{0}^{*}, \mathbb{U}_{+}^{2}$, in addition to $\mathbb{S}_{1}^{2}$, in the same order.

Suppose $\beta=\beta(s)$ is the unit speed spacelike curve with timelike principal normal in $\mathbb{E}_{1}^{3}$, and suppose $\kappa(s)$ and $\tau(s)$ are the natural curvature and torsion of $\beta(s)$, in the same order. Suppose $\left\{\zeta_{1}(s), \zeta_{2}(s), \zeta_{3}(s)\right\}$ is a Serret-Frenet frame related to the curve $\beta(s)$, then the Serret-Frenet formulas read

$$
\left(\begin{array}{l}
\zeta_{1}^{\prime} \\
\zeta_{2}^{\prime} \\
\zeta_{3}^{\prime}
\end{array}\right)=\left(\begin{array}{lll}
0 & \kappa & 0 \\
\kappa & 0 & \tau \\
0 & \tau & 0
\end{array}\right)\left(\begin{array}{l}
\zeta_{1} \\
\zeta_{2} \\
\zeta_{3}
\end{array}\right)=\psi \times\left(\begin{array}{l}
\zeta_{1} \\
\zeta_{2} \\
\zeta_{3}
\end{array}\right)
$$

where $\psi(s)=\tau \zeta_{1}-\kappa \zeta_{3}$ is the Darboux vector of the Serret-Frenet frame. At this study, $\beta^{\prime}(s)$ denotes the derivatives of $\beta$ respecting to arc length parameter. The Serret-Frenet vector fields satisfy the following relations:

$$
\begin{aligned}
& \left\langle\zeta_{1}, \zeta_{1}\right\rangle=\left\langle\zeta_{3}, \zeta_{3}\right\rangle=1,\left\langle\zeta_{2}, \zeta_{2}\right\rangle=-1, \\
& \zeta_{1} \times \zeta_{2}=\zeta_{3}, \zeta_{1} \times \zeta_{3}=\zeta_{2}, \zeta_{2} \times \zeta_{3}=\zeta_{1} .
\end{aligned}
$$

Definition 1. The moving pseudoorthogonal frame $\left\{\xi_{1}, \xi_{3}, \xi_{3}\right\}$, along the nonnull space curve $\alpha(s)$, is rotation minimizing frame $(\mathrm{RMF})$ respecting to $\xi_{1}$ in case its angular velocity $\omega$ insures $\left\langle\omega, \xi_{1}\right\rangle=0$ or, similarly, the derivatives of $\xi_{2}$ and $\xi_{3}$ are both parallel to $\xi_{1}$. Analogously, characterization holds when $\xi_{2}$ or $\xi_{3}$ is selected to be the reference direction.

Using Definition 1, it is observed that the Serret-Frenet frame is RMF respecting to the principal normal $\xi_{2}$ but not 
respecting to the tangent $\zeta_{1}$ and the binormal $\zeta_{3}$. Even though the Serret-Frenet frame is not RMF respecting to $\zeta_{1}$, it is easy to derive similar RMF from it. New normal plane vectors $\left(\mathbf{N}_{1}, \mathbf{N}_{2}\right)$ are determined along the rotation of $\left(\zeta_{2}, \zeta_{3}\right)$ as

$$
\left(\begin{array}{c}
\mathbf{T}_{1} \\
\mathbf{N}_{1} \\
\mathbf{N}_{2}
\end{array}\right)=\left(\begin{array}{ccc}
1 & 0 & 0 \\
0 & \cosh \theta & \sinh \theta \\
0 & \sinh \theta & \cosh \theta
\end{array}\right)\left(\begin{array}{l}
\zeta_{1} \\
\zeta_{2} \\
\zeta_{3}
\end{array}\right),
$$

with a certain Lorentzian timelike angle $\theta(s) \geq 0$. The set $\left\{\mathbf{T}_{1}\right.$, $\left.\mathbf{N}_{1}, \mathbf{N}_{2}\right\}$ is called RMF or Bishop frame. The RMF vector insure the following relations:

$$
\begin{aligned}
& \left\langle\mathbf{T}_{1}, \mathbf{T}_{1}\right\rangle=\left\langle\mathbf{N}_{2}, \mathbf{N}_{2}\right\rangle=1,\left\langle\mathbf{N}_{1}, \mathbf{N}_{1}\right\rangle=-1, \\
& \mathbf{T}_{1} \times \mathbf{N}_{1}=\mathbf{N}_{2}, \mathbf{T}_{1} \times \mathbf{N}_{2}=\mathbf{N}_{1}, \mathbf{N}_{1} \times \mathbf{N}_{2}=\mathbf{T}_{1} .
\end{aligned}
$$

Therefore, the Bishop frame reads

$$
\left(\begin{array}{c}
\mathbf{T}_{1}^{\prime} \\
\mathbf{N}_{1}^{\prime} \\
\mathbf{N}_{2}^{\prime}
\end{array}\right)=\left(\begin{array}{ccc}
0 & \kappa_{1} & \kappa_{2} \\
\kappa_{1} & 0 & 0 \\
-\kappa_{2} & 0 & 0
\end{array}\right)\left(\begin{array}{c}
\mathbf{T}_{1} \\
\mathbf{N}_{1} \\
\mathbf{N}_{2}
\end{array}\right)=\tilde{\boldsymbol{\omega}} \times\left(\begin{array}{c}
\mathbf{T}_{1} \\
\mathbf{N}_{1} \\
\mathbf{N}_{2}
\end{array}\right),
$$

where $\widetilde{\omega}(s)=-\kappa_{2} \mathbf{N}_{1}-\kappa_{1} \mathbf{N}_{2}$ is the Bishop Darboux vector. Here, the Bishop curvatures are determined as $\kappa_{1}(s)=\kappa \cosh \vartheta$ and $\kappa_{2}(s)=-\kappa \sinh \vartheta$. Comparing equations (5) and (9), it is observed that the relative velocity is

$$
\psi(s)-\widetilde{\boldsymbol{\omega}}(s)=\tau \zeta_{1} .
$$

One can show that

$$
\begin{aligned}
& \kappa_{1}^{2}-\kappa_{2}^{2}=\kappa^{2}, \text { and } \vartheta=-\tanh ^{-1}\left(\frac{\kappa_{2}}{\kappa_{1}}\right) ; \kappa_{1} \neq 0, \\
& \theta(s)=-\int_{s_{0}}^{s} \tau d s+\theta_{0}, \theta_{0}=\theta(0) .
\end{aligned}
$$

As a consequence, both Serret-Frenet frame and RMF identical iff $\beta(s)$ is the planar, which means $\tau=0$.

The spacelike vector is defined as

$$
\boldsymbol{\omega}(s)=-\frac{\kappa_{2}}{\kappa_{1}} \mathbf{N}_{1}-\mathbf{N}_{2},
$$

and we name it the modified Bishop Darboux vector through $\beta(s)$. A Bishop spherical Darboux image $\mathbf{e}(s): I \longrightarrow$ $\mathbb{S}_{1}^{2}(0,1)$ is defined by

$$
\mathbf{e}(s)=\frac{\boldsymbol{\omega}(s)}{\|\boldsymbol{\omega}(s)\|}=\frac{-\kappa_{1}}{\sqrt{\kappa_{1}^{2}-\kappa_{2}^{2}}}\left(\frac{\kappa_{2}}{\kappa_{1}} \mathbf{N}_{1}+\mathbf{N}_{2}\right) .
$$

Therefore, we consider a new geometric invariant $\rho(s)=\kappa_{2} \kappa_{1}^{\prime}-\kappa_{1} \kappa_{2}^{\prime}$.

Definition 2. The sweeping surface through $\beta(s)$ is the surface given as

$$
\begin{aligned}
M: \mathbf{R}(s, u) & =\boldsymbol{\beta}(s)+F(s) \mathbf{x}(u) \\
& =\boldsymbol{\alpha}(s)+x_{1}(u) \mathbf{N}_{1}(s)+x_{2}(u) \mathbf{N}_{2}(s),
\end{aligned}
$$

where $\beta(s)$ named the (at least $C^{1}$-continuous) spine curve, $0 \leq s \leq T$, and $s$ is the arc length parameter. $\mathbf{x}(u)$ is the planar profile (cross-section) curve defined as parametric presentation, $\mathbf{x}(u)=\left(0 x_{1}(u), x_{2}(u)\right)^{T}$, “T” refers to transposition, and $u \in I \subseteq \mathbb{R}$. The semiorthogonal matrix $F(s)=\left\{\mathbf{T}_{1}(s)\right.$, $\mathbf{N}_{1}(s), \mathbf{N}_{2}(s)$ \} specifies the RMF through $\beta(s)$.

Kinematically, the sweeping surface $\mathbf{R}(s, u)$ is generating by the moving of the profile curve $\mathbf{x}(u)$ through the spine curve $\beta(s)$ with the orientation as introduced by $F(s)$. The profile curve $\mathbf{x}(u)$ is in the $2 \mathrm{D}$ or $3 \mathrm{D}$ space that passes through the spine curve $\beta(s)$ during sweeping. Interestingly, $\mathrm{RMF}$ allows for a simple characterization of spine curve.

Definition 3. The surface at Minkowski 3-space $\mathbb{E}_{1}^{3}$ named the timelike surface in case the induced metric at the surface is the Lorentz metric and also it named the spacelike surface in case the induced metric at the surface is a positive definite Riemannian metric, which means the normal vector on spacelike (timelike) surface is the timelike (spacelike) vector.

\section{Spacelike Sweeping Surface and Its Singularities}

We present the spacelike sweeping surface at Minkowski 3space $\mathbb{E}_{1}^{3}$. Consider the planar profile (cross-section) that is defined as $\mathbf{x}(u)=(0, \cosh u, \sinh u)$. Using equation (14), we obtain

$$
M: \mathbf{R}(s, u)=\boldsymbol{\beta}(s)+\cosh u \mathbf{N}_{1}+\sinh u \mathbf{N}_{2} .
$$

Using equation (9) resulted in

$$
\left.\begin{array}{l}
\mathbf{R}_{u}(s, u)=\sinh u \mathbf{N}_{1}+\cosh u \mathbf{N}_{2}, \\
\mathbf{R}_{s}(s, u)=\left(1+\kappa_{1} \cosh u-\kappa_{2} \sinh u\right) \mathbf{T}_{1} .
\end{array}\right\} .
$$

The unit normal vector of $M$ is

$$
\mathbf{N}(s, u)=\frac{\mathbf{R}_{s} \times \mathbf{R}_{u}}{\left\|\mathbf{R}_{s} \times \mathbf{R}_{u}\right\|}=\cosh u \mathbf{N}_{1}+\sinh u \mathbf{N}_{2} .
$$

The main aim of this study is given in the following theorem:

Theorem 1. Suppose $\beta: I \longrightarrow \mathbb{E}_{1}^{3}$ is the unit speed spacelike curve with a timelike principal normal, and $\kappa_{1}^{2}-\kappa_{2}^{2} \neq 0$. Then, for any fixed $\mathbf{x} \in \mathbb{S}_{1}^{2}(0,1)$, one has the following:

A.

(1) The image of Bishop spherical Darboux is locally diffeomorphic to the line $\{0\} \times \mathbb{R}$ at $s_{0}$ iff $\rho\left(s_{0}\right) \neq 0$

(2) The image of Bishop spherical Darboux is locally diffeomorphic to a cusp $C \times \mathbb{R}$ at $s_{0}$ if $\rho\left(s_{0}\right)=0$, as well $\rho^{\prime}\left(s_{0}\right) \neq 0$

B.

(1) $M$ is locally diffeomorphic to cuspidal edge CE at $\left(s_{0}, u_{0}\right)$ iff $\mathbf{x}= \pm e\left(s_{0}\right)$, as well $\rho\left(s_{0}\right) \neq 0$

(2) $M$ is locally diffeomorphic to swallowtail $S W$ at $\left(s_{0}, u_{0}\right)$ iff $\mathbf{x}= \pm e\left(s_{0}\right), \rho\left(s_{0}\right)=0$, as well $\rho^{\prime}\left(s_{0}\right)=0$ 
Here, $\quad C \times \mathbb{R}=\left\{\left(x_{1}, x_{2}\right) \mid x_{1}^{2}=x_{2}^{3}\right\} \times \mathbb{R}, \quad C E=\left\{\left(x_{1}, x_{2}\right.\right.$, $\left.\left.x_{3}\right) \mid x_{1}=u, x_{2}=v^{2}, x_{3}=v^{3}\right\}, \quad$ and $\quad S W=\left\{\left(x_{1}, x_{2}\right.\right.$, $\left.\left.x_{3}\right) \mid x_{1}=u, x_{2}=3 v^{2}+u v^{2}, x_{3}=4 v^{3}+2 u v\right\}$. The pictures of $C \times \mathbb{R}, C E$, and $S W$ are shown in Figures $1-3$.

3.1. Lorentzian Bishop Height Functions. We will introduce two different families of Lorentzian Bishop height functions that will be useful to study singularities of $M$ as follows [1-3]: $H: I \times \mathbb{S}_{1}^{2} \longrightarrow \mathbb{R}$, by $H(s, \mathbf{x})=\langle\beta(s), \mathbf{x}\rangle$. It is called Lorentzian Bishop height function. The notation $h_{\mathbf{x}}(s)=H(s, \mathbf{x})$ will be used for all fixed $\mathbf{x} \in \mathbb{S}_{1}^{2}$. In addition, it is defined $\widetilde{H}: I \times \mathbb{S}_{1}^{2} \times \mathbb{R} \longrightarrow \mathbb{R}$, using $\widetilde{H}(s, \mathbf{x}, w)=$ $\langle\beta, \mathbf{x}\rangle-w$. It is called extended Lorentzian Bishop height function of $\beta(s)$. It is denoted that $\widetilde{h}_{\mathbf{x}}(s)=\widetilde{H}(s, \mathbf{x})$. From here, parameter $s$ will not be written.

We have the following proposition:

Proposition 1. Let $\beta: I \longrightarrow \mathbb{E}_{1}^{3}$ be the unit speed spacelike curve with a timelike principal normal, with $\kappa_{1}^{2}-\kappa_{2}^{2} \neq 0$. Then,

A.

(1) $h_{\mathbf{x}}^{\prime}(s)=0$ iff $\mathbf{x}=a_{1} \mathbf{N}_{1}+a_{2} \mathbf{N}_{2}$, and $-a_{1}^{2}+a_{2}^{2}=1$.

(2) $h_{\mathbf{x}}^{\prime}(s)=h_{x}^{\prime \prime}(s)=0$ iff $\mathbf{x}= \pm e(s)$

(3) $h_{\mathbf{x}}^{\prime}(s)=h_{x}^{\prime \prime}(s)=h_{\mathbf{x}} \prime \prime \prime(s)=0$ iff $\mathbf{x}= \pm e(s)$, and $\rho(s)=0$

(4) $h_{\mathbf{x}}^{\prime}(s)=h_{x}^{\prime \prime}(s)=h_{\mathbf{x}} \prime \prime \prime(s)=h_{\mathbf{x}}^{(4)}(s)=0$ iff $\mathbf{x}= \pm e(s)$, and $\rho(s)=\rho^{\prime}(s)=0$

(5) $h_{\mathbf{x}}^{\prime}(s)=h_{x}^{\prime \prime}(s)=h_{\mathbf{x}} \prime \prime \prime(s)=h_{\mathbf{x}}^{(4)}(s)=h_{\mathbf{x}}^{(5)}(s)=0 \quad$ iff $\mathbf{x}= \pm e(s)$, and $\rho(s)=\rho^{\prime}(s)=\rho^{\prime \prime}(s)=0$

$B$.

(1) $\tilde{h}_{\mathbf{x}}(s)=0$ iff there is $\langle\beta, \mathbf{x}\rangle=w$

(2) $\tilde{h}_{\mathbf{x}}(s)=\widetilde{h}_{x}^{\prime}(s)=0$ if there are $a_{1}, a_{2} \in \mathbb{R}$, that is, $\mathbf{x}=\cosh u \mathbf{N}_{1}+\sinh u \mathbf{N}_{2}$, and $\langle\beta, \mathbf{x}\rangle=w$

(3) $\tilde{h}_{\mathbf{x}}(s)=\widetilde{h}_{\mathbf{x}}^{\prime}(s)=\widetilde{h}_{x}^{\prime \prime}(s)=\tilde{h}_{x}^{\prime \prime}(s)=0$ iff $\mathbf{x}= \pm \mathbf{e}(s)$, $\langle\beta, \mathbf{x}\rangle=w$, and $\rho(s)=0$

(4) $\tilde{h}_{\mathbf{x}}(s)=\tilde{h}_{\mathbf{x}}^{\prime}(s)=\widetilde{h}_{x}^{\prime \prime}(s)=\tilde{h}_{x}^{\prime \prime}(s)=\widetilde{h}_{x}^{\prime \prime}(s)=0 \quad$ iff $\mathbf{x}= \pm \mathbf{e}(s),\langle\beta, \mathbf{x}\rangle=w$, and $\rho(s)=\rho^{\prime}(s)=0$

(5) $\widetilde{h}_{\mathbf{x}}(s)=\widetilde{h}_{\mathbf{x}}^{\prime}(s)=\widetilde{h}_{x}^{\prime \prime}(s)=\widetilde{h}_{x}^{\prime \prime}(s)=\widetilde{h}_{x}^{\prime \prime}(s)=\tilde{h}_{x}^{(4)}(s)=0$ iff $\mathbf{x}= \pm \mathbf{e}(s),\langle\beta, \mathbf{x}\rangle=w$ and $\rho(s)=\rho^{\prime}(s)=$ $\rho^{\prime \prime}(s)=0$

(6) $\widetilde{h}_{\mathbf{x}}(s)=\widetilde{h}_{\mathbf{x}}^{\prime}(s)=\widetilde{h}_{x}^{\prime \prime}(s)=\widetilde{h}_{x}^{\prime \prime}(s)=\widetilde{h}_{x}^{\prime \prime}(s)=\widetilde{h}_{x}^{(4)}(s)=$ $\tilde{h}_{x}^{(4)}(s)=0 \quad$ iff $\quad \mathbf{x}= \pm \mathbf{e}(s), \quad\langle\beta, \mathbf{x}\rangle=w$, and $\rho(s)=\rho^{\prime}(s)=\rho^{\prime \prime}(s)=\rho \prime \prime \prime(s)=0$

Proof. Using equation (9) results in that $\left\|\mathbf{T}_{1}^{\prime}\right\|^{2} \neq 0$ iff $\kappa_{1}^{2}-\kappa_{2}^{2} \neq 0$.

A.

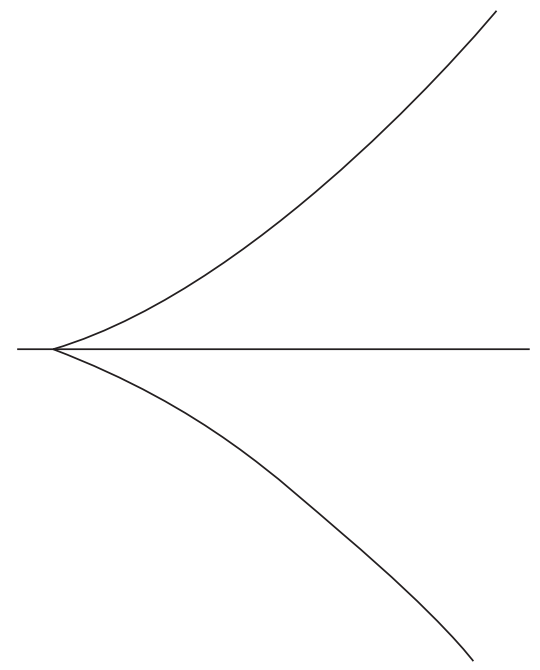

Figure 1: $C \times \mathbb{R}$.

(1) Because $h_{\mathbf{x}}^{\prime}(s)=\left\langle\mathbf{T}_{1}, \mathbf{x}\right\rangle$ and $\left\{\mathbf{T}_{1}, \mathbf{N}_{1}, \mathbf{N}_{2}\right\}$ is RMF through $\beta(s)$, then there are $a_{1}, a_{2} \in \mathbb{R}$ such that $\mathbf{x}=a_{1} \mathbf{N}_{1}+a_{2} \mathbf{N}_{2}$. Because of $\mathbf{x} \in \mathbb{S}_{1}^{2}$, we have $-a_{1}^{2}+a_{2}^{2}=1$. The opposite holds as well.

(2) Because $h_{\mathbf{x}}^{\prime \prime}(s)=\left\langle\mathbf{T}_{1}^{\prime}, \mathbf{x}\right\rangle=\left\langle\kappa_{1} \mathbf{N}_{1}+\kappa_{2} \mathbf{N}_{2}, \mathbf{x}\right\rangle=0$, we have that $-a_{1} \kappa_{1}+a_{2} \kappa_{2}=0$. It follows from the fact $-a_{1}^{2}+a_{2}^{2}=1$ that $a_{1}=\mp \kappa_{2} / \sqrt{\kappa_{1}^{2}-\kappa_{2}^{2}}$ and $a_{2}= \pm \kappa_{1} / \sqrt{\kappa_{1}^{2}-\kappa_{2}^{2}}$. Therefore, we obtain

$\mathbf{x}=\left(\mp \frac{\kappa_{1}}{\sqrt{\kappa_{1}^{2}-\kappa_{2}^{2}}}\left(\frac{\kappa_{2}}{\kappa_{1}} \mathbf{N}_{1}+\mathbf{N}_{2}\right)\right)(s)= \pm \mathbf{e}(s)$.

Therefore, $h_{\mathbf{x}}^{\prime}(s)=h_{x}^{\prime \prime}(s)=0$ iff $\mathbf{x}= \pm e(s)$.

(3) Because $\quad h_{\mathbf{x}} \prime \prime \prime(s)=\left\langle\mathbf{T}_{1}^{\prime \prime}, \mathbf{x}\right\rangle=\left\langle\left(\kappa_{1}^{2}-\kappa_{2}^{2}\right) \mathbf{T}_{1}+\right.$ $\kappa_{1}^{\prime} \mathbf{N}_{1}+\kappa_{2}^{\prime} \mathbf{N}_{2}, \mathbf{x}>=0$, using conditions of (2), we obtain

$\pm \frac{\kappa_{1}}{\sqrt{\kappa_{1}^{2}-\kappa_{2}^{2}}}\left(\frac{\kappa_{2} \kappa_{1}^{\prime}-\kappa_{1} \kappa_{2}^{\prime}}{\kappa_{1}}\right)(s)= \pm \frac{\kappa_{1}}{\sqrt{\kappa_{1}^{2}-\kappa_{2}^{2}}}\left(\frac{\rho}{\kappa_{1}}\right)(s)=0$.

Thus, $h_{\mathbf{x}}^{\prime}(s)=h_{x}^{\prime \prime}(s)=h_{\mathbf{x}} \prime \prime \prime(s)=0$ iff $\mathbf{x}= \pm e(s)$, and $\rho(s)$.

(4) Since

$$
\left.\begin{array}{rl}
h_{\mathbf{x}}^{(4)}(s)= & <\mathbf{T}_{1}^{\prime \prime \prime}, \mathbf{x}>=<3\left(\kappa_{1} \kappa_{1}^{\prime}-\kappa_{2} \kappa_{2}^{\prime}\right) \mathbf{T}_{1}+\left(\kappa_{1}^{\prime \prime}+\kappa_{1}\left(\kappa_{1}^{2}-\kappa_{2}^{2}\right)\right) \mathbf{N}_{1} \\
& +\left(\kappa_{2}^{\prime \prime}+\kappa_{2}\left(\kappa_{1}^{2}-\kappa_{2}^{2}\right)\right) \mathbf{N}_{2}, \quad \mathbf{x}>=0
\end{array}\right\}
$$




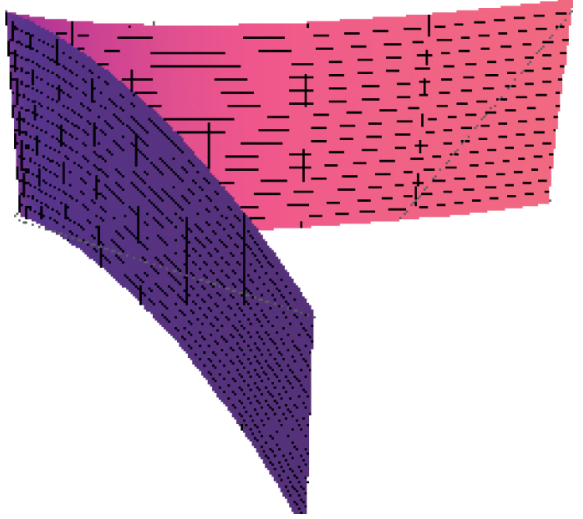

Figure 2: CE.

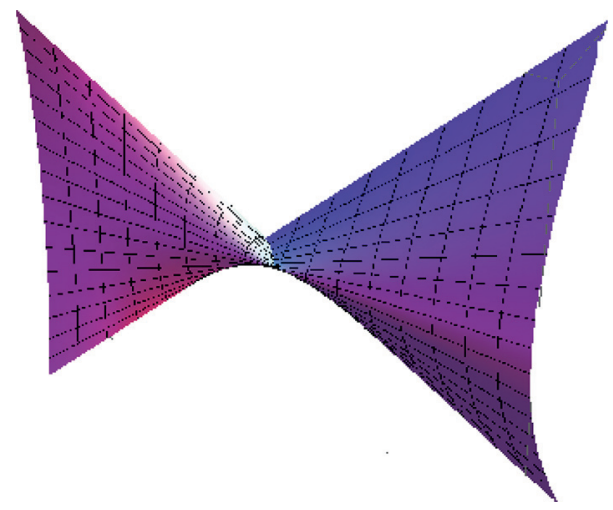

Figure 3: SW.

by the conditions of (3), we have that

$\pm \frac{\kappa_{1}}{\sqrt{\kappa_{1}^{2}-\kappa_{2}^{2}}}\left(\frac{\left(\kappa_{2} \kappa_{1}^{\prime}-\kappa_{1} \kappa_{2}^{\prime}\right)^{\prime}}{\kappa_{1}}\right)(s)= \pm \frac{1}{\sqrt{\kappa_{1}^{2}-\kappa_{2}^{2}}} \rho^{\prime}(s)=0$.

Thus, $\quad h_{\mathbf{x}}^{\prime}(s)=h_{x}^{\prime \prime}(s)=h_{\mathbf{x}}^{\prime \prime}(s)=h_{\mathbf{x}}^{(4)}(s)=0 \quad$ iff $\mathbf{x}= \pm e(s)$, and $\rho(s)=\rho^{\prime}(s)=0$.

(5) Since $h_{\mathbf{x}}^{(5)}(s)=<\mathbf{T}_{1}^{(4)}, \mathbf{x}>0$, we have

$$
\left.\begin{array}{l}
<\left(\left(\kappa_{1}^{2}-\kappa_{2}^{2}\right)^{2}+4\left(\kappa_{2} \kappa_{2}^{\prime \prime}-\kappa_{1} \kappa_{1}^{\prime \prime}\right)+3\left(\kappa_{1}^{\prime 2}-\kappa_{2}^{\prime 2}\right)\right) \mathbf{T}_{1} \\
+\left(\kappa_{1}^{\prime \prime}+5 \kappa_{1}\left(\kappa_{1}^{\prime} \kappa_{1}-\kappa_{2}^{\prime} \kappa_{2}\right)+\kappa_{2}^{\prime} \kappa_{1}^{2}-\kappa_{1}^{\prime} \kappa_{2}^{2}\right) \mathbf{N}_{1} \\
+\left(\kappa_{2}^{\prime \prime}+5 \kappa_{2}\left(\kappa_{2}^{\prime} \kappa_{2}-\kappa_{1}^{\prime} \kappa_{1}\right)+\kappa_{1}^{\prime} \kappa_{2}^{2}-\kappa_{2}^{\prime} \kappa_{1}^{2}\right) \mathbf{N}_{2}, \mathbf{x}>=0
\end{array}\right\}
$$

By using the conditions of (4), we have

$$
\pm \frac{1}{\sqrt{\kappa_{1}^{2}-\kappa_{2}^{2}}}\left(\frac{\kappa_{1} \kappa_{2}^{\prime \prime \prime}-\kappa_{2} \kappa_{1}^{\prime \prime \prime}+\left(\kappa_{2} \kappa_{1}^{\prime}-\kappa_{1} \kappa_{2}^{\prime}\right)\left(\kappa_{1}^{2}-\kappa_{2}^{2}\right)}{\kappa_{1}}\right)=0 .
$$

Therefore, $\quad h_{\mathbf{x}}^{\prime}(s)=h_{x}^{\prime \prime}(s)=h_{\mathbf{x}^{\prime}}^{\prime \prime}(s)=h_{\mathbf{x}}^{(4)}(s)=h_{\mathbf{x}}^{(5)}=0$ iff $\mathbf{x}= \pm e(s)$, as well $\rho(s)=\rho^{\prime}(s)=\rho^{\prime \prime}(s)=0$.

B

Using similar computation as in proof of $A$, we obtain B (1).

Proposition 2. Suppose $\beta: I \longrightarrow \mathbb{E}_{1}^{3}$ is the unit speed spacelike curve with the timelike principal normal, and $\kappa_{1}^{2}-\kappa_{2}^{2}>0$. Then, we have $\rho(s)=0$ iff

$$
\mathbf{e}(s)=\frac{-\kappa_{1}}{\sqrt{\kappa_{1}^{2}-\kappa_{2}^{2}}}\left(\frac{\kappa_{2}}{\kappa_{1}} \mathbf{N}_{1}+\mathbf{N}_{2}\right)
$$

is a constant vector.

Proof. Let $\kappa_{1}^{2}-\kappa_{2}^{2} \neq 0$. Using simple calculations, we have that

$$
\mathbf{e}^{\prime}(s)=\frac{\rho(s)}{\left(\sqrt{\kappa_{1}^{2}-\kappa_{2}^{2}}\right)^{3}}\left(\kappa_{1} \mathbf{N}_{1}+\kappa_{2} \mathbf{N}_{2}\right)
$$

Thus, $\mathbf{e}^{\prime}(s)=0$ iff $\rho(s)=\kappa_{2} \kappa_{1}^{\prime}-\kappa_{1} \kappa_{2}^{\prime}=0$.

Proposition 3. Suppose $\beta: I \longrightarrow \mathbb{E}_{1}^{3}$ is the unit speed spacelike curve with the timelike principal normal, and $\kappa_{1}^{2}-\kappa_{2}^{2}>0$. Then, it is stated that

(a) $\beta$ is the B-slant helix iff $\kappa_{2} / \kappa_{1}$ is a constant

(b) $\mathbf{N}_{2}$ is the part of circle at $\mathbb{S}_{1}^{2}$, and its center is the spacelike constant vector $\mathbf{e}_{0}$.

Proof. (a) Lets have $\rho(s)=\kappa_{2} \kappa_{1}^{\prime}-\kappa_{1} \kappa_{2}^{\prime}=0$. Hence, we can write

$$
\left(\frac{\kappa_{2}}{\kappa_{1}}\right)^{\prime}=\frac{\kappa_{1} \kappa_{2}^{\prime}-\kappa_{2} \kappa_{1}^{\prime}}{\kappa_{1}^{2}}=\frac{-\rho(s)}{\kappa_{1}^{2}}=0 .
$$

This means that $\kappa_{2} / \kappa_{1}=$ constant, such that, $\beta$ is the B-slant helix.

(b) Suppose that $\kappa_{1}^{2}-\kappa_{2}^{2}>0$. Since

$$
\begin{aligned}
\left\langle e, N_{2}\right\rangle & =\frac{-\kappa_{1}}{\sqrt{\kappa_{1}^{2}-\kappa_{2}^{2}}}\left\langle\left(\frac{\kappa_{2}}{\kappa_{1}} N_{1}+N_{2}\right), N_{2}\right\rangle \\
& =\frac{-1}{\sqrt{1-\kappa_{2}^{2} / \kappa_{1}^{2}}}=\text { constant. }
\end{aligned}
$$

In other words, $N_{2}$ is the part of circle at $\mathbb{S}_{1}^{2}$, and its center is the constant spacelike vector $\mathbf{e}_{0}(s)$.

3.2. Unfolding of Functions by One-Variable. We use some general results at the singularity theory for families of function germs [1-3]. Suppose $F:\left(\mathbb{R} \times \mathbb{R}^{r},\left(s_{0}, \mathbf{x}_{0}\right)\right) \longrightarrow \mathbb{R}$ is the smooth function, and $f(s)=F_{x_{0}}\left(s, \mathbf{x}_{0}\right)$. Therefore, $F$ 
named the $r$-parameter unfolding of $f(s)$. It is said that $f(s)$ has $\mathrm{A}_{k}$ singularity at $s_{0}$ in case $f^{(p)}\left(s_{0}\right)=0$ for every $1 \leq p \leq k$, and $f^{(k+1)}\left(s_{0}\right) \neq 0$. Additionally, $f$ has $\mathrm{A}_{\geqslant k}$ singularity $(k \geqslant 1)$ at $s_{0}$. Suppose the $(k-1)$ jet of the partial derivative $\partial F / \partial x_{i}$ at $s_{0}$ is $j^{(k-1)}\left(\partial F / \partial x_{i}\left(s, \mathbf{x}_{0}\right)\right)\left(s_{0}\right)=$ $\sum_{j=0}^{k-1} L_{j i}\left(s-s_{0}\right)^{j}$ (without the constant term), for $i=1, \ldots, r$. Therefore, $F(s)$ named the $p$ versal unfolding in case of the $k \times r$ matrix of coefficients $\left(L_{j i}\right)$ from the rank $k(k \leq r)$. So, we write an important set about the unfolding relative to the previous notations. The discriminant set of $F$ is

$$
\mathfrak{D}_{F}=\left\{\mathbf{x} \in \mathbb{R}^{r} \mid \text { there exists } s \text { with } F(s, \mathbf{x})=\frac{\partial F}{\partial s}(s, \mathbf{x})=0 \text { at }(s, \mathbf{x})\right\} .
$$

The bifurcation set of $F$ is

$$
\mathfrak{B}_{F}=\left\{\mathbf{x} \in \mathbb{R}^{r} \mid \text { there exists } s \text { with } \frac{\partial F}{\partial s}(s, \mathbf{x})=\frac{\partial^{2} F}{\partial s^{2}}(s, \mathbf{x})=0 \text { at }(s, \mathbf{x})\right\} .
$$

Similar to [1-3], we state the following theorem:

Theorem 2. Suppose $F:\left(\mathbb{R} \times \mathbb{R}^{r},\left(s_{0}, \mathbf{x}_{0}\right)\right) \longrightarrow \mathbb{R}$ is the $r$ parameter unfolding of $f(s)$, with $A_{k}$ singularity at $s_{0}$.

Considering $F$ is the $p$ versal unfolding,

(a) In case $k=1, \mathfrak{D}_{F}$ is locally diffeomorphic to $\{0\} \times \mathbb{R}^{r-1}$, and $\mathfrak{B}_{F}=\varnothing$;

(b) In case $k=2, \mathfrak{D}_{F}$ is locally diffeomorphic to $\mathbf{C} \times \mathbb{R}^{r-2}$, and $\mathfrak{B}_{F}$ is locally diffeomorphic to $\{0\} \times \mathbb{R}^{r-1}$;

(c) In case $k=3, \mathfrak{D}_{F}$ is locally diffeomorphic to $\mathbf{S W} \times \mathbb{R}^{r-3}$, and $\mathfrak{B}_{F}$ is locally diffeomorphic to $\mathbf{C} \times \mathbb{R}^{r-2}$.

Hence, we have the following proposition:

Proposition 4. Suppose $\beta: I \longrightarrow \mathbb{E}_{1}^{3}$ is the unit speed spacelike curve with the timelike principal normal, and $\kappa_{1}^{2}-\kappa_{2}^{2} \neq 0$.

(1) In case $h_{\mathbf{x}}(s)=H(s, \mathbf{x})$ has the $A_{k}$ singularity $(k=2,3)$ at $s_{0} \in \mathbb{R}$, and $H$ is the $p$ versal unfolding of $h_{\mathbf{x}_{0}}\left(s_{0}\right)$
(2) In case $\tilde{h}_{\mathbf{x}}(s)=\widetilde{H}(s, \mathbf{x}, w)$ has the $A_{k}$-singularity $(k=2,3)$ at $s_{0} \in \mathbb{R}$, and $\tilde{H}$ is the p-versal unfolding of $\widetilde{h}_{\mathbf{x}_{0}}\left(s_{0}\right)$

Proof.

(1) Since $\mathbf{x}=\left(x_{0}, x_{1}, x_{2}\right) \in \mathbb{S}_{1}^{2},-x_{0}^{2}+x_{1}^{2}+x_{2}^{2}=1, x_{0}, x_{1}$, and $x_{2}$ cannot be all zero. Without the loss of generality, suppose $x_{2} \neq 0$. Then, by $x_{2}=\sqrt{1+x_{0}^{2}-x_{1}^{2}}$, we have

$$
H(s, \mathbf{x})=-x_{0} \beta_{0}(s)+x_{1} \beta_{1}(s)+\sqrt{1+x_{0}^{2}-x_{1}^{2}} \beta_{2}(s) .
$$

Thus, we have that

$$
\left.\begin{array}{c}
\frac{\partial H}{\partial x_{0}}=-\beta_{0}(s)+\frac{x_{0} \beta_{2}(s)}{\sqrt{1+x_{0}^{2}-x_{1}^{2}}}, \frac{\partial H}{\partial x_{1}}=\beta_{1}(s)-\frac{x_{1} \beta_{2}(s)}{\sqrt{1+x_{1}^{2}-x_{2}^{2}}}, \\
\frac{\partial^{2} H}{\partial s \partial x_{0}}=-\beta_{0}^{\prime}(s)+\frac{x_{0} \beta_{2}^{\prime}(s)}{\sqrt{1+x_{0}^{2}-x_{1}^{2}}}, \frac{\partial^{2} H}{\partial s \partial x_{1}}=\beta_{1}^{\prime}(s)-\frac{x_{1} \beta_{2}^{\prime}(s)}{\sqrt{1+x_{1}^{2}-x_{2}^{2}}} .
\end{array}\right\}
$$


Therefore, the 2 jets of $\partial H / \partial x_{i}$ at $s_{0}(i=0,1)$ are as follows: let $\mathbf{x}_{0}=\left(x_{00}, x_{10}, x_{20}\right) \in \mathbb{S}_{1}^{2}$, and assume $x_{20} \neq 0$, then

$$
\left.\begin{array}{r}
j^{1}\left(\frac{\partial H}{\partial x_{0}}\left(s, \mathbf{x}_{0}\right)\right)=\left(-\beta_{0}^{\prime}(s)+\frac{x_{00} \beta_{2}^{\prime}(s)}{x_{20}}\right)\left(s-s_{0}\right), \\
j^{1}\left(\frac{\partial H}{\partial x_{1}}\left(s, \mathbf{x}_{0}\right)\right)=\left(\beta_{1}^{\prime}(s)-\frac{x_{10} \beta_{2}^{\prime}(s)}{x_{20}}\right)\left(s-s_{0}\right),
\end{array}\right\} .
$$

(i) In case $h_{\mathbf{x}_{0}}\left(s_{0}\right)$ has the $A_{2}$ singularity at $s_{0}$, then $h_{\mathbf{x}_{0}}^{\prime}\left(s_{0}\right)=0$. So, the $(2-1) \times 2$ matrix of coefficients $\left(L_{j i}\right)$ is

$$
A=\left(-\beta_{0}^{\prime}(s)+\frac{x_{00} \beta_{2}^{\prime}(s)}{x_{20}} \beta_{1}^{\prime}(s)-\frac{x_{10} \beta_{2}^{\prime}(s)}{x_{20}}\right) .
$$

If the matrix $A$ has rank equal zero, then

$$
\beta_{0}^{\prime}(s)=\frac{x_{00} \beta_{2}^{\prime}(s)}{x_{20}}, \beta_{1}^{\prime}(s)=\frac{x_{10} \beta_{2}^{\prime}(s)}{x_{20}} .
$$

Since $\left\|\beta^{\prime}\left(s_{0}\right)\right\|=\left\|\mathbf{T}_{1}\left(s_{0}\right)\right\|=1$, we have $\beta_{2}^{\prime}\left(s_{0}\right) \neq 0$, so that we have the contradiction as follows:

$$
\begin{aligned}
0 & =\left\langle\left(\beta_{0}^{\prime}\left(s_{0}\right), \beta_{1}^{\prime}\left(s_{0}\right), \beta_{2}^{\prime}\left(s_{0}\right)\right),\left(x_{00}, x_{10}, x_{20}\right)\right\rangle \\
& =-\beta_{0}^{\prime}\left(s_{0}\right) x_{00}+\beta_{1}^{\prime}\left(s_{0}\right) x_{10}+\beta_{2}^{\prime}\left(s_{0}\right) x_{20} \\
& =-\frac{x_{00}^{2} \beta_{2}^{\prime}\left(s_{0}\right)}{x_{20}}+\frac{x_{10}^{2} \beta_{2}^{\prime}\left(s_{0}\right)}{x_{20}}+\beta_{2}^{\prime}\left(s_{0}\right) x_{20} \\
& =\frac{\beta_{2}^{\prime}\left(s_{0}\right)}{x_{20}}\left(-x_{00}^{2}+x_{10}^{2}+x_{20}^{2}\right) \\
& =\frac{\beta_{2}^{\prime}\left(s_{0}\right)}{x_{20}} \neq 0 .
\end{aligned}
$$

Then, $\operatorname{rank}(A)=1$, as well $H$ is the $p$ versal unfolding of $h_{\mathbf{x}_{0}}$ at $s_{0}$.

(ii) In case $h_{\mathbf{x}_{0}}\left(s_{0}\right)$ has the $A_{3}$ singularity at $s_{0} \in \mathbb{R}$, then $h_{\mathbf{x}_{0}}^{\prime}\left(s_{0}\right)=h_{\mathbf{x}_{0}}^{\prime \prime}\left(s_{0}\right)=0$, and using Proposition 1 ,

$$
\mathbf{e}\left(s_{0}\right)=\frac{\kappa_{1}}{\sqrt{\kappa_{1}^{2}-\kappa_{2}^{2}}}\left(\frac{\kappa_{2}}{\kappa_{1}} \mathbf{N}_{1}+\mathbf{N}_{2}\right),
$$

where $\kappa_{1}^{2}-\kappa_{2}^{2}>0, \rho^{\prime}\left(s_{0}\right)=0$, and $\rho^{\prime \prime}\left(s_{0}\right) \neq 0$. So the $(3-1) \times 2$ matrix of the coefficients $\left(L_{j i}\right)$ is

$$
B=\left(\begin{array}{cc}
L_{11} & L_{12} \\
L_{21} & L_{22}
\end{array}\right)=\left(\begin{array}{cc}
-\beta_{0}^{\prime}(s)+\frac{x_{00} \beta_{2}(s)}{x_{20}} & \beta_{1}^{\prime}(s)-\frac{x_{10} \beta_{2}^{\prime}(s)}{x_{20}} \\
-\beta_{0}^{\prime \prime}+\frac{x_{00} \beta_{2}^{\prime \prime}(s)}{x_{20}} & \beta_{1}^{\prime \prime}(s)-\frac{x_{10} \beta_{2}^{\prime \prime}(s)}{x_{20}}
\end{array}\right) .
$$

It is required that the $2 \times 2$ matrix $B$ is a nonsingular matrix. Clearly, this matrix determinate at $s_{0}$ is

$$
\begin{aligned}
\operatorname{det}(B) & =\frac{1}{x_{20}}\left|\begin{array}{ccc}
-\beta_{0}^{\prime} & \beta_{1}^{\prime} & \beta_{2}^{\prime} \\
-\beta_{0}^{\prime \prime} & \beta_{1}^{\prime \prime} & \beta_{2}^{\prime \prime} \\
x_{00} & x_{10} & x_{20}
\end{array}\right| \\
& =\frac{1}{x_{20}}\left\langle\boldsymbol{\beta}^{\prime} \times \boldsymbol{\beta}^{\prime \prime}, \mathbf{e}_{0}\right\rangle \\
& =\mp \frac{\kappa_{1}}{x_{20} \sqrt{\kappa_{1}^{2}-\kappa_{2}^{2}}}\left\langle\boldsymbol{\beta}^{\prime} \times \boldsymbol{\beta}^{\prime \prime},\left(\frac{\kappa_{2}}{\kappa_{1}} \mathbf{N}_{1}+\mathbf{N}_{2}\right)\right\rangle .
\end{aligned}
$$

Since $\beta^{\prime}=\mathbf{T}_{1}$, we have $\beta^{\prime \prime}=\kappa_{1} \mathbf{N}_{1}+\kappa_{2} \mathbf{N}_{2}$. Substituting these relations to the previous equality, we obtain

$$
\operatorname{det}(B)=\mp \frac{\sqrt{\kappa_{1}^{2}-\kappa_{2}^{2}}}{x_{20}} \neq 0
$$


which resulted in $\operatorname{rank}(B)=2$.

(2) Using similar notations as in (1), we get

$\tilde{H}\left(s, \mathbf{x}, x_{2}\right)=-x_{0} \beta_{0}(s)+x_{1} \beta_{1}(s)+\sqrt{1+x_{0}^{2}-x_{1}^{2}} \beta_{2}(s)-x_{2}$.

We require the $2 \times 3$ matrix,

$$
G=\left(\begin{array}{ccc}
-\beta_{0}^{\prime}(s)+\frac{x_{00} \beta_{2}(s)}{x_{20}} & \beta_{1}^{\prime}(s)-\frac{x_{10} \beta_{2}^{\prime}(s)}{x_{20}}-1 \\
-\beta_{0}^{\prime \prime}+\frac{x_{00} \beta_{2}^{\prime \prime}(s)}{x_{20}} & \beta_{1}^{\prime \prime}(s)-\frac{x_{10} \beta_{2}^{\prime \prime}(s)}{x_{20}} & 0
\end{array}\right) \text {, }
$$

to have the maximal rank. Using case (1) in equation (38), the second raw of $G$ will not be zero; then, $\operatorname{rank}(G)=2$.

Proof of Theorem 1. (1) Using Proposition 1, the bifurcation set of $H(s, \mathbf{x})$ is

$$
\mathfrak{B}_{H}=\left\{\frac{\kappa_{1}}{\sqrt{\kappa_{1}^{2}-\kappa_{2}^{2}}}\left(\frac{\kappa_{2}}{\kappa_{1}} \mathbf{N}_{1}+\mathbf{N}_{2}\right)|s \in \mathbb{R}| s \in \mathbb{R}\right\} .
$$

The assertion (1) of Theorem 1 follows from Propositions 1 and 4 and Theorem 2 . The discriminant set of $\widetilde{H}(s, \mathbf{x})$ is written as

$$
\mathfrak{D}_{\widetilde{H}}=\left\{\mathbf{x}_{0}=\boldsymbol{\beta}+\cosh u \mathbf{N}_{1}+\sinh u \mathbf{N}_{2} \mid s \in \mathbb{R}\right\} .
$$

The assertion (1) of Theorem 1 follows from Propositions 1 and 4 and Theorem 2 .

Example 1. Consider this spacelike helix:

$$
\boldsymbol{\alpha}(s)=\left(\frac{\sqrt{3}}{2} \sinh s, \frac{s}{2}, \frac{\sqrt{3}}{2} \cosh s\right), \quad-1 \leq s \leq 1 .
$$

It is easy to show that

$$
\begin{aligned}
& \zeta_{1}(s)=\left(\frac{\sqrt{3}}{2} \sinh s, \frac{1}{2}, \frac{\sqrt{3}}{2} \cosh s\right), \\
& \zeta_{2}(s)=(\sinh s, 0, \cosh s) \\
& \zeta_{3}(s)=\left(\frac{1}{2} \cosh s,-\frac{\sqrt{3}}{2}, \frac{1}{2} \sinh s\right), \\
& \kappa(s)=\frac{\sqrt{3}}{2}, \text { and } \tau(s)=\frac{1}{2} .
\end{aligned}
$$

Taking $\theta_{0}=0$, we obtain $\theta(s)=-1 / 2 s$. By the use of equation (9), we have

$$
\kappa_{1}(s)=\cosh \frac{s}{2} \text {, and } \kappa_{2}(s)=-\sinh \frac{s}{2} .
$$

Hence, the geometric invariant is

$$
\rho(s)=\frac{1}{2} .
$$

Therefore, the transformation matrix is written as

$$
\left(\begin{array}{c}
\mathbf{T}_{1} \\
\mathbf{N}_{1} \\
\mathbf{N}_{2}
\end{array}\right)=\left(\begin{array}{ccc}
1 & 0 & 0 \\
0 & \cosh \frac{s}{2} & -\sinh \frac{s}{2} \\
0 & -\sinh \frac{s}{2} & \cosh \frac{s}{2}
\end{array}\right)\left(\begin{array}{l}
\zeta_{1} \\
\zeta_{2} \\
\zeta_{3}
\end{array}\right) .
$$

From this, we have

$$
\begin{aligned}
& \mathbf{N}_{1}=\left(\begin{array}{l}
N_{11} \\
N_{12} \\
N_{13}
\end{array}\right)=\left(\begin{array}{c}
\cosh \frac{s}{2} \sinh s-\frac{1}{2} \sinh \frac{s}{2} \cosh s \\
\frac{\sqrt{3}}{2} \sinh \frac{s}{2} \\
\cosh \frac{s}{2} \cosh s-\frac{1}{2} \sinh \frac{s}{2} \sinh s
\end{array}\right) \\
& \mathbf{N}_{2}=\left(\begin{array}{c}
N_{21} \\
N_{22} \\
N_{23}
\end{array}\right)=\left(\begin{array}{c}
\frac{1}{2} \cosh \frac{s}{2} \cosh s-\sinh \frac{s}{2} \sinh s \\
-\frac{1}{2} \cosh \frac{s}{2} \\
\frac{1}{2} \cosh \frac{s}{2} \sinh s-\sinh \frac{s}{2} \cosh s
\end{array}\right) .
\end{aligned}
$$

Hence, the Bishop spherical Darboux image (Figure 4)

$$
\mathbf{e}(s)=\sinh \frac{s}{2}\left(\begin{array}{c}
N_{11} \\
N_{12} \\
N_{13}
\end{array}\right)-\cosh \frac{s}{2}\left(\begin{array}{c}
N_{21} \\
N_{22} \\
N_{23}
\end{array}\right) .
$$

The spacelike sweeping surface (Figure 5) is

$$
\begin{aligned}
M: \mathbf{R}(s, u)= & \left(\frac{\sqrt{3}}{2} \sinh s, \frac{s}{2}, \frac{\sqrt{3}}{2} \cosh s\right)+\cosh u\left(\begin{array}{c}
N_{11} \\
N_{12} \\
N_{13}
\end{array}\right) \\
& +\sinh u\left(\begin{array}{c}
N_{21} \\
N_{22} \\
N_{23}
\end{array}\right) .
\end{aligned}
$$




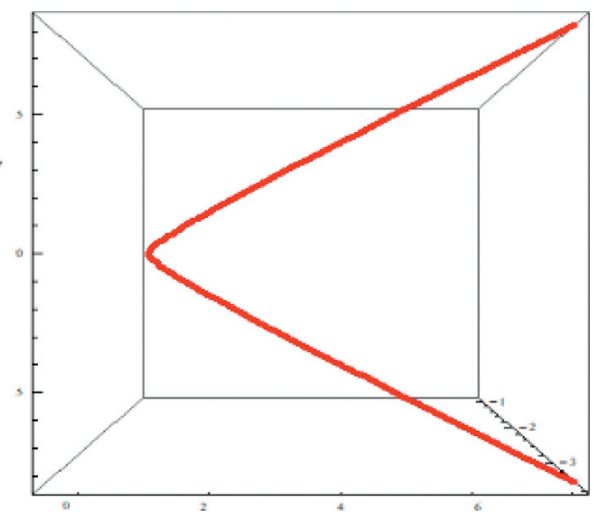

Figure 4: Bishop spherical Darboux image has a cusp as $s=0$.

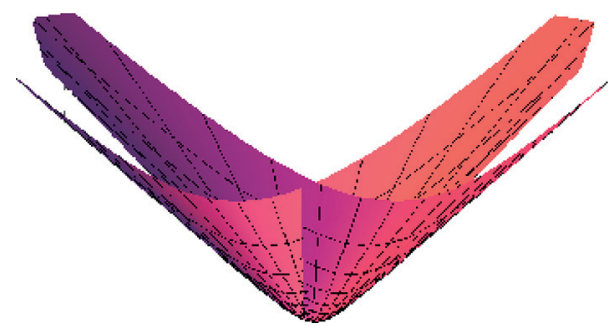

Figure 5: Spacelike sweeping surface with spacelike helix singularity curve.

3.3. Singularities of Developable Surfaces. It is known that the developable surface is a special case of ruled surface. This kind of surfaces is used extensively in manufacture of automobile body parts, airplane wings, as well some ship parts. As a result, we analyze the case that the profile curve $\mathbf{x}$ degenerates to the line. Therefore, we have the next spacelike developable surface:

$$
M: \mathbf{Q}(s, u)=\boldsymbol{\beta}(s)+u \mathbf{N}_{2}(s), \quad u \in \mathbb{R} .
$$

Moreover, we have the following timelike developable surface:

$$
M^{\perp}: \mathbf{Q}^{\perp}(s, u)=\boldsymbol{\beta}(s)+u \mathbf{N}_{1}(s), \quad u \in \mathbb{R} .
$$

Clearly, $\mathbf{Q}(s, 0)=\alpha(s)$ (respectively, $\mathbf{Q}^{\perp}(s, 0)=\alpha(s)$ ), $0 \leq s \leq L$, such that, the surface $M$ (respectively, $M^{\perp}$ ) interpolates the curve $\alpha(s)$. We also have

$$
M: \mathbf{Q}_{s} \times \mathbf{Q}_{u}=\left(1-u \kappa_{2}\right) \mathbf{N}_{1}(s),
$$

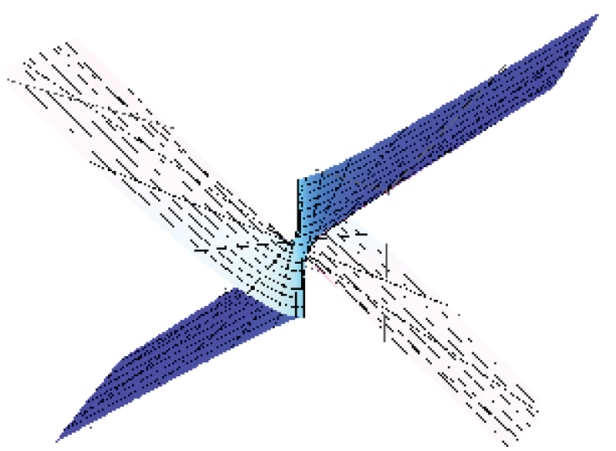

Figure 6: The timelike developable surface.

as well

$$
M^{\perp}: \mathbf{Q}_{s}^{\perp} \times \mathbf{Q}_{u}^{\perp}=\left(1+u \kappa_{1}\right) \mathbf{N}_{1}(s) .
$$

Therefore, we obtain $M$ (respectively, $M^{\perp}$ ) that is nonsingular at $\left(s_{0}, u_{0}\right)$ if and only if $1-u_{0} \kappa_{2}\left(s_{0}\right) \neq 0$ (respectively $\left.\left(1+u_{0} \kappa_{1}\left(s_{0}\right) \neq 0\right)\right)$. Hence, we can classify the singularities of developable surface $M$ by using $\kappa_{2}$.

Theorem 3. Suppose $M$ is the spacelike developable given as in equation (53). Therefore, we have

(1) $M$ is locally diffeomorphic to cuspidal edge at $\left(s_{0}, u_{0}\right)$ iff $\kappa_{2}\left(s_{0}\right)=0$ and $\kappa_{2}^{\prime}\left(s_{0}\right) \neq 0$

(2) $M$ is locally diffeomorphic to swallowtail at $\left(s_{0}, u_{0}\right)$ iff $\kappa_{2}\left(s_{0}\right) \neq 0$ and $\kappa_{2}^{\prime}\left(s_{0}\right) / \kappa_{2}^{2}\left(s_{0}\right) \neq 0$

Proof. If there is a parameter $s_{0}$, that is, $\kappa_{2}\left(s_{0}\right)=0$, and $u_{0}^{\prime}=$ $\kappa_{2}^{\prime}\left(s_{0}\right) / \kappa_{2}^{2}\left(s_{0}\right) \neq 0\left(\kappa_{2}^{\prime}\left(s_{0}\right) \neq 0\right), M$ is locally diffeomorphic to the cuspidal edge at $\left(s_{0}, u_{0}\right)$. As a result, assertion (1) holds. In addition, in case there is a parameter $s_{0}$, that is, $u_{0}=1 / \kappa_{2}\left(s_{0}\right) \neq 0, u_{0}^{\prime}=\kappa_{2}^{\prime}\left(s_{0}\right) / \kappa_{2}^{2}\left(s_{0}\right)=0$, and $\left(1 / \kappa_{2}\left(s_{0}\right)\right)^{\prime \prime} \neq$ 0 , and $M$ is locally diffeomorphic to swallowtail at $\left(s_{0}, u_{0}\right)$, assertion (2) holds.

Example 2. By making using of Example 1, we have

(1) If $s_{0}=0$, then $\kappa_{2}\left(s_{0}\right)=0$ and $\kappa_{2}^{\prime}\left(s_{0}\right) \neq 0$. The timelike developable surface

$$
M: \mathbf{Q}(s, u)=\left(\frac{\sqrt{3}}{2} \sinh s, \frac{s}{2}, \frac{\sqrt{3}}{2} \cosh s\right)+u\left(\begin{array}{c}
\frac{1}{2} \cosh \frac{s}{2} \cosh s-\sinh \frac{s}{2} \sinh s \\
-\frac{\sqrt{3}}{2} \cosh \frac{s}{2} \\
\frac{1}{2} \cosh \frac{s}{2} \sinh s-\sinh \frac{s}{2} \cosh s
\end{array}\right)
$$




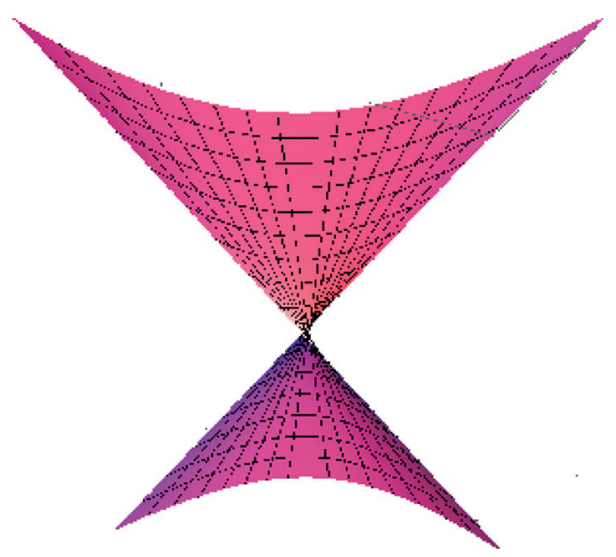

Figure 7: The spacelike developable surface.

where $u \in \mathbb{R}$ is locally diffeomorphic to the cuspidal edge (Figure 6).

(2) In case $s_{0}=0, \kappa_{1}\left(s_{0}\right) \neq 0$ and $\kappa_{2}^{\prime}\left(s_{0}\right)=0$. The spacelike developable surface is

$$
M^{\perp}: \mathbf{Q}^{\perp}(s, u)=\left(\frac{\sqrt{3}}{2} \sinh s, \frac{s}{2}, \frac{\sqrt{3}}{2} \cosh s\right)+u\left(\begin{array}{c}
\cosh \frac{s}{2} \sinh s-\frac{1}{2} \sinh \frac{s}{2} \cosh s \\
\frac{\sqrt{3}}{2} \sinh \frac{s}{2} \\
\cosh \frac{s}{2} \cosh s-\frac{1}{2} \sinh \frac{s}{2} \sinh s
\end{array}\right)
$$

where $u \in \mathbb{R}$ is locally diffeomorphic to swallowtail (Figure 7).

\section{Conclusion}

In this study, we introduced the notion of spacelike sweeping surfaces with rotation minimizing frames at Minkowski 3space $\mathbb{E}_{1}^{3}$. Using the singularity, we classified the generic properties and present a new geometric invariant related to the singularities of this spacelike sweeping surface. It leads to that the generic singularities of this sweeping surface are cuspidal edge and swallowtail, and the types of these singularities can be characterized by this geometric invariant, in the same order. Subsequently, we derived the sufficient and necessary conditions for the surface to be developable ruled surfaces. At the end, some examples are introduced in order to clarify the theoretical results.

\section{Data Availability}

No data were used to support this study.

\section{Conflicts of Interest}

The authors declare that they have no conflicts of interest.

\section{Acknowledgments}

This research was funded by the Deanship of Scientific Research at Princess Nourah Bint Abdulrahman University through the Fast-track Research Funding Program.

\section{References}

[1] J. W. Bruce and P. J. Giblin, "Generic geometry," The American Mathematical Monthly, vol. 90, no. No. 8, pp. 529-545, 1983.

[2] J. W. Bruce and P. J. Giblin, Curves and Singularities, Cambridge University Press, Cambridge, UK, 2nd edition, 1992.

[3] R. Cipolla and P. J. Giblin, Visual Motion of Curves and Surfaces, Cambridge Univ. Press, Cambridge, UK, 2000.

[4] K. Saji, M. Umehara, and K. Yamada, "The geometry of fronts," Annals of Mathematics, vol. 169, no. 2, pp. 491-529, 2009.

[5] Y. Kitagawa and M. Umehara, "Extrinsic diameter of immersed flat tori in S 3," Geometriae Dedicata, vol. 155, no. 1, pp. 105-140, 2011.

[6] K. Teramoto, "Parallel and dual surfaces of cuspidal edges," Differential Geometry and Its Applications, vol. 44, pp. 52-62, 2016.

[7] S. Izumiya and N. Takeuchi, "Singularities of ruled surfaces in 3," Mathematical Proceedings of the Cambridge Philosophical Society, vol. 130, no. 1, pp. 1-11, 2001. 
[8] M. P. Do Carmo, Differential Geometry of Curves and Surface, Prentice-Hall, Englewood Cliffs, NJ, USA, 1976.

[9] Z. Xu, R. Feng, and J.-G. Sun, "Analytic and algebraic properties of canal surfaces," Journal of Computational and Applied Mathematics, vol. 195, no. 1-2, pp. 220-228, 2006.

[10] S. Izumiya, K. Saji, and N. Takeuchi, "Circular surfaces," Advances in Geometry, vol. 7, no. 2, pp. 295-313, 2007.

[11] L. Cui, D. Wang, and J. S. Dai, "Kinematic geometry of circular surfaces with a fixed radius based on Euclidean invariants," Journal of Mechanical Design, vol. 131, no. 10, 2009.

[12] R. A. Abdel-Baky and Y. Unlütürk, "On the curvatures of spacelike circular surfaces," Kuwait Journal of Science, vol. 43, no. 3, pp. 50-58, 2016.

[13] R. A. Abdel-Baky, N. Alluhaibi, A. Ali, and F. Mofarreh, "A study on timelike circular surfaces in Minkowski 3-space," International Journal of Geometric Methods in Modern Physics, vol. 17, no. 6, Article ID 2050074, 2020.

[14] M. Fouad Naghi and R. A. Abdel-Baky, "Timelike sweeping surfaces and singularities," International Journal of Geometric Methods in Modern Physics, vol. 18, no. 1, Article ID 2150006, 2021.

[15] Ö. Köse, "A method of the determination of a developable ruled surface," Mechanism and Machine Theory, vol. 34, no. 8, pp. 1187-1193, 1999.

[16] H. Pottmann and J. Wallner, "Approximation algorithms for developable surfaces," Computer Aided Geometric Design, vol. 16, no. 6, pp. 539-556, 1999.

[17] H. Zhao and G. Wang, "A new method for designing a developable surface utilizing the surface pencil through a given curve," Progress in Natural Science, vol. 18, no. 1, pp. 105-110, 2008.

[18] O. G. Yildız, S. O. Karakus, and H. H. Hacisalihoglu, "On the determination of a developable spherical orthotomic ruled surface," Bulletin of Mathematical Sciences, vol. 5, pp. 137146, 2015.

[19] R. A. Abdel-Baky, "Developable surfaces through sweeping surfaces," Bulletin of the Iranian Mathematical Society, vol. 45, no. 1, pp. 951-963, 2019.

[20] R. L. Bishop, "There is more than one way to frame a curve," The American Mathematical Monthly, vol. 82, no. 3, pp. 246-251, 1975.

[21] B. Bükcu and M. K. Karacan, "On the slant helices according to Bishop frame of the timelike curve in Lorentzian space," Tamkang Journal of Mathematics, vol. 39, no. 3, pp. 255-262, 2008.

[22] M. Grbovic and E. Nešovic, "On the Bishop frames of pseudo null and null Cartan curves in Minkowski 3-space," Journal of Mathematical Analysis and Applications, vol. 461, pp. 219233, 2018.

[23] O. Keskin and Y. Yayli, "An application of N-Bishop frame to spherical images for direction curves," International Journal of Geometric Methods in Modern Physics, vol. 14, no. 11, Article ID 1750162, 2017.

[24] B. O’Neil, Semi-Riemannian Geometry Geometry, with Applications to Relativity, Academic Press, New York, NY, USA, 1983.

[25] J. Walfare, "Curves and surfaces in minkowski space," Ph.D. Thesis, Faculty of Science, Leuven, Belgium, 1995. 